\title{
O gerenciamento dos resíduos sólidos dos serviços de saúde no contexto da saúde ambiental
}

A gestão segura e sustentável dos resíduos sólidos dos serviços de saúde (RSSS) é um imperativo de saúde pública. A gestão inadequada dos RSSS constitui um risco significativo para os pacientes, trabalhadores da saúde, da comunidade e do meio ambiente. Este é um estudo que objetivou realizar um levantamento bibliográfico a respeito dos resíduos sólidos dos serviços de saúde (RSSS) e a saúde ambiental. Realizouse um levantamento nas bases de dados eletrônicas SCIELO, LILACS, BIREME, BVS e portal de periódicos CAPES. Este artigo realiza uma revisão de literatura abordando os aspectos históricos, conceituais, institucionais e legais dos RSSS, enfocando a problemática do gerenciamento inadequado e a importância do gerenciamento dos RSSS na ótica da saúde ambiental. A complexidade do gerenciamento dos RSSS exige uma análise inter e transdisciplinar e o conhecimento sobre variáveis relacionadas com diferentes áreas do conhecimento. Diante dos resultados encontrados até agora, é fundamental o comprometimento dos atores envolvidos no processo de gerenciamento dos resíduos apoiando-se na vontade política dos governantes, na disponibilidade de recursos humanos e econômicos, são fatores necessários para uma gestão eficaz, a qual permitirá a comunidade uma melhoria em diversos setores primordiais, entre eles: saúde pública e saúde ambiental.

Palavras-chave: Resíduos de Serviços de Saúde; Saúde Ambiental; Gerenciamento de Resíduos.

\section{Aste management of health services in the environmental context}

\begin{abstract}
Safe and sustainable management of health care solid waste (SSR) is a public health imperative. Inadequate management of SSRIs poses a significant risk to patients, health workers, the community and the environment. This is a study that aimed to carry out a bibliographical survey on solid waste from health services (SSR) and environmental health. A survey was carried out in the electronic databases SCIELO, LILACS, BIREME, VHL and CAPES journal portal. This article presents a review of literature addressing the historical, conceptual, institutional and legal aspects of SSRs, focusing on the problem of inadequate management and the importance of SSH management in environmental health. The complexity of RSSS management requires inter- and trans-disciplinary analysis and knowledge about variables related to different areas of knowledge. In view of the results found so far, it is essential that the stakeholders involved in the waste management process, based on the political will of the government, the availability of human and economic resources, are necessary factors for effective management, which will allow the community An improvement in several key sectors, including: public health and environmental health.
\end{abstract}

Keywords: Health Service Waste; Environmental Health; Waste Management.

Topic: Epidemiologia e Saúde Ambiental

Reviewed anonymously in the process of blind peer.

Dayane Clock

Instituto Federal de Santa Catarina, Brasil. http://lattes.cnpq.br/1716874607640720 dclockluiz@gmail.com

Therezinha Maria Novais de Oliveira Universidade da Região de Joinville, Brasil. http://lattes.cnpq.br/8358410394755408 tnovais@univille.br
Received: $10 / 10 / 2016$

Approved: 12/01/2017

\section{Referencing this:}

CLOCK, D.; OLIVEIRA, T. M. N.. O gerenciamento dos resíduos sólidos dos serviços de saúde no contexto da saúde ambiental. Revista lberoAmericana de Ciências Ambientais, v.8, n.2, p.73-84, 2017. DOI: http://doi.org/10.6008/SPC2179-6858.2017.002.0006 


\section{INTRODUÇÃO}

Atualmente, a abordagem de questões da interface saúde e meio ambiente têm se tornado uma temática importante para pesquisadores de diversas áreas do conhecimento, ao possibilitar a análise e a intervenção para a manutenção da vida no Planeta. Embora a relação entre saúde e meio ambiente se faça mais presente, quando da divulgação de situações e eventos catastróficos e ameaçadores ou, quando dados alarmantes sobre a degradação ambiental são divulgados, percebe-se cada vez mais a necessidade de se consolidar um corpo de conhecimentos científicos que permita evidenciar as implicações inerentes a este contexto, especialmente no que tange a saúde humana (CAMPONOGARA et al., 2008).

A problemática dos Resíduos Sólidos dos Serviços de Saúde (RSSS), como qualquer outra que vem colaborando para a agressão ao meio ambiente, parece suscitar a emergência de uma nova postura ética, de renovação de valores, cidadania, compromisso social, num entendimento de que tudo faz parte da grande teia da vida, implicando uma nova consciência, de responsabilidade e comprometimento, em nossas ações, no nosso agir, na nossa forma de perceber e de viver e conviver nesse ambiente, que nos constitui e que constituímos (CORREA et al., 2005).

Em especial, no contexto da assistência em saúde, esta aproximação é marcada pelo viés normativo, visivelmente relacionado ao gerenciamento de resíduos sólidos, sem uma discussão mais ampla sobre a relação entre a assistência à saúde e a questão ecológica. Assim, os profissionais da saúde, sob este enfoque, são direcionados, predominantemente, para o cumprimento de normas, comprometendo o desenvolvimento de uma visão mais ampla sobre o tema.

Ao contrário de outros segmentos empresariais, que já avançaram no tema, a área da saúde necessita de iniciativas que contribuam para uma nova realidade na qual os estabelecimentos de atenção à saúde sejam numa visão holística realmente promotores de saúde e não apenas gestores de doenças e a preocupação com a sustentabilidade seja um dos caminhos para manutenção e melhoria da qualidade de vida das pessoas (NAIME et al., 2007).

Na concepção de Schneider (2004), os RSSS representam uma ameaça à saúde pública e ao meio ambiente não somente pela sua quantidade, mas pelo alto potencial de risco de propagação de doenças e, sendo assim, o seu gerenciamento necessita de maior segurança no manejo, proporcionando, ao mesmo tempo, a melhor qualidade dos serviços prestados e incentivando a redução do volume de resíduos produzidos.

Tratar a saúde de forma integrada com os fatores ambientais e as questões econômicas traduz-se na busca da qualidade da saúde ambiental, a qual, necessariamente, está ligada ao desenvolvimento de processos ecologicamente sustentáveis (SCHNEIDER, 2004).

A qualidade de vida depende da qualidade do ambiente, portanto, a negligência em termos de gestão e gerenciamento de resíduos de serviços de saúde pode contribuir para a poluição do meio ambiente e afetar a saúde dos seres humanos. $\mathrm{O}$ desafio dos hospitais no que diz respeito à promoção e desenvolvimento da saúde é reconhecer que os determinantes básicos da saúde são os cuidados de saúde em conjunto com a 
qualidade do ambiente, considerando-se que o meio ambiente tem um papel fundamental em relação à saúde pública das populações (RAMíREZ, 2012).

De acordo com Ramos (2013), o comprometimento dos atores envolvidos no processo de gerenciamento dos resíduos apoiando-se na vontade política de nossos governantes e por fim, na disponibilidade de recursos humanos e econômicos, seriam então, fatores necessários para uma gestão eficaz, a qual permitiria a comunidade uma melhoria em diversos setores primordiais, entre eles: saúde pública e saúde ambiental.

Diante do exposto, o gerenciamento de resíduo sólido de serviços de saúde é uma necessidade urgente e incontestável e requerem, das autoridades competentes, e dos atores envolvidos no processo, providências no sentido de minimizar este desequilíbrio no setor de saúde ambiental (CARVALHO, 2010).

\section{REVISÃO TEÓRICA}

\section{Abordagem Metodológica}

Este é um artigo de revisão de abordagem qualitativa no conceito de Minayo (2004), a pesquisa qualitativa, responde a questões muito particulares, pois se preocupa, nas Ciências Sociais, com um nível de realidade que não pode ser quantificado, ou seja, trabalha com o universo de significados, motivos, crenças, valores e atitudes, o que corresponde a um espaço mais profundo das relações, dos processos e dos fenômenos que não podem ser reduzidos à operacionalização de variáveis. De caráter bibliográfico, conforme orientam Lüdke e André (2010) e onde são expressos os aspectos teóricos do tema em apreço, numa análise crítica do material estudado.

Nesse contexto, realizou-se um levantamento bibliográfico em bases de dados eletrônicas sendo definidos descritores de assuntos visando uma consulta mais direcionada aos resíduos de serviço de saúde e a saúde ambiental. As bases de dados pesquisadas foram, SCIELO, LILACS, BIREME, BVS e portal de periódicos CAPES. As pesquisas eletrônicas aconteceram no período de março a julho de 2015.

Durante o processo de busca foram utilizados descritores, resíduos de serviços de saúde, resíduos sólidos de serviços de saúde (RSSS), gerenciamento de resíduos, gerenciamento de resíduos sólidos de serviços de saúde, legislação sanitária, legislação em saúde, saúde ambiental e saúde e meio ambiente.

Os descritores mais amplos foram associados a outros mais restritos visando direcionar a pesquisa dos materiais. Procedeu-se a leitura dos resumos dos artigos e teses encontrados e estes foram enquadrados em três categorias: legislação frente aos RSSS e experiências frente ao gerenciamento dos RSSS. A pesquisa da legislação também foi realizada diretamente das leis e estas foram agrupadas na categoria legislação frente aos RSSS.

\section{Aspectos históricos, conceituais, institucionais e legais dos RSSS}

No Brasil, até há pouco tempo, os atuais resíduos de serviços de saúde eram conhecidos somente como "lixo hospitalar", uma vez que, a história da gestão dos resíduos de serviço de saúde se confunde com 
a história da institucionalização dos hospitais. Essa denominação foi substituída mais tarde pela atual ao se verificar que não só os hospitais, mas também outros estabelecimentos prestadores de serviços na área de saúde geravam resíduos com características semelhantes (CAMPOS, et al. 2014).

A assistência hospitalar no Brasil surgiu no século XVI, com as Irmandades de Misericórdias, as Santas Casas, semelhantes àquelas fundadas em Lisboa em 1498 por Dona Leonor de Lencastre. Acredita-se que a primeira Santa Casa de Misericórdia criada no país seja a de Santos, fundada em 1543 por Brás Cubas. Fundada numa época que Santos não era sequer uma vila, o então Hospital de Todos os Santos deu origem ao próprio nome da cidade (PUGLIESI, 2010).

Com a institucionalização e o início da assistência hospitalar, houve o início da geração de Resíduos de Serviços de Saúde (RSS) e a preocupação com o assunto não é recente, conforme Schneider (2004) há estudos datados de 1903 que expressam a preocupação devido ao caráter infeccioso dos resíduos hospitalares que contaminaram pessoas pelo contato direto na manipulação.

No Brasil, a produção científica e bibliográfica relacionada ao então denominado "resíduo hospitalar" tem seu primeiro marco em 1969, com a comunicação "Lixo de hospitais" de autoria de R. Zaltman na Revista Lixo e limpeza pública, da então Faculdade de Higiene e Saúde Pública da USP. Em sequência, no ano de 1972, Luz e Guimarães publicam o artigo "Resíduos hospitalares" na Revista Saúde Pública, no qual apresentam informações diversas sobre os "resíduos hospitalares" em estabelecimentos norte-americanos: volumes produzidos, formas de acondicionamento no local de produção, de transporte interno, de armazenamento para a coleta, de remoção e de destinação final (PUGLIESI, 2010).

Historicamente, os fatos marcantes no que tange aos RSS do final do século XIX até os dias de hoje, foi à instalação do primeiro incinerador em um hospital nos Estados Unidos em 1891, no município de Nova lorque. No Brasil, o primeiro incinerador foi construído em Manaus, em 1896. A incineração começou a ganhar importância no início do século XX, mais precisamente na década de 40 , quando foram publicados vários trabalhos sobre este método de tratamento (RISSO, 1993).

No Brasil em 29 de dezembro de 1951, o Estado de São Paulo edita a lei estadual n¹561-A na qual estabeleceu, em seu artigo 195, que todos os hospitais deveriam possuir sistema de coleta de lixo que oferecesse garantias de higiene e de assepsia, e que o lixo resultante dos serviços médicos cirúrgicos deveria ser incinerado. No município de São Paulo, em $1^{\circ}$ de junho de 1963, a lei municipal nº.297 determinou que deveriam dispor de incineradores todos os hospitais, sanatórios, casas de saúde, maternidades, ambulatórios, creches e outros. A lei não chegou a ser regulamentada, não foram especificados exatamente quais os resíduos que deveriam ser incinerados, nem estabelecidos requisitos mínimos para os incineradores. Como decorrência, as tentativas de sua aplicação revelaram-se infrutíferas e inviáveis (LUZ \& GUIMARÃES, 1972).

Em 1970 a incineração torna-se obrigatória no Estado de São Paulo em função de um decreto estadual. O Decreto Estadual 52.497 aprova o regulamento a que se refere o artigo 22 do decreto-lei 211 do mesmo ano, o qual estabeleceu, em seu artigo 128, que "é obrigatória a instalação de incineração do lixo séptico ou cirúrgico em incinerador localizado no perímetro do hospital" (LUZ \& GUIMARÃES, 1972). 
Até então as orientações eram voltadas para aspectos sanitários externos - etapas de coleta e tratamento dos resíduos, mais focados no destino final dos resíduos- não sendo considerados ainda os aspectos sanitários internos - relacionados ao manejo interno dos resíduos nos geradores (PUGLIESI, 2010). Mais recentemente o problema recebeu um aporte significativo nas pesquisas, discussões e legislações relativas ao gerenciamento dos RSSS, devido principalmente ao grande desenvolvimento ocorrido nos estudos no campo da infecção hospitalar e do meio ambiente a partir do final da década de 80 (Pugliesi, 2010).

O quadro1, elaborado por Pugliesi (2010), apresenta uma divisão histórica da evolução dos RSS. Observa-se nele que as questões relacionadas com o manejo interno dos resíduos pelos estabelecimentos geradores são tratadas somente a partir das últimas décadas do século XX, refletindo a incorporação nas legislações das práticas de controle de infecção hospitalar assim como a inclusão de princípios do direito como poluidor-pagador e responsabilidade compartilhada.

Quadro 1: Resumo histórico dos principais acontecimentos relacionados aos RSS.

\begin{tabular}{|c|c|}
\hline Período & Resumo Histórico \\
\hline 1881 & Instalação do primeiro incinerador em um hospital dos Estados Unidos. \\
\hline 1903 & $\begin{array}{l}\text { Publicado trabalho que apresenta a relação existente entre a contração de doenças e pessoas que } \\
\text { manipulam resíduos hospitalares. }\end{array}$ \\
\hline $1930-1939$ & $\begin{array}{l}\text { Começa a ser dada importância aos problemas produzidos pelos resíduos sólidos em hospitais e buscam- } \\
\text { se soluções para alguns deles. }\end{array}$ \\
\hline 1940-1949 & $\begin{array}{l}\text { Numerosos trabalhos são publicados sobre a utilização de incineradores como método de tratamento, } \\
\text { sua importância, bem como suas desvantagens. }\end{array}$ \\
\hline 1950-1959 & $\begin{array}{l}\text { Destaca-se a urgente necessidade de manejo apropriado dos resíduos hospitalares de forma a evitar que } \\
\text { se transformem em fonte de contaminação. A incineração passa a ser utilizada e começa a preocupação } \\
\text { com o controle radioativo. }\end{array}$ \\
\hline $1960-1969$ & $\begin{array}{l}\text { É dada maior ênfase à problemática desse tipo de resíduos, bem como às possíveis soluções através de } \\
\text { publicações tratando dos temas: controle adequado que evite a dispersão de doenças infecciosas; } \\
\text { necessidade de mecanização dos serviços; o acondicionamento em sacos plásticos; equipamentos } \\
\text { utilizados na redução de volume e controle dos líquidos decorrentes dessas operações; utilização de } \\
\text { incineradores e os problemas de poluição atmosférica; tratamento dos resíduos provenientes de } \\
\text { indivíduos com doenças contagiosas; os problemas originados da utilização de materiais radioativos. }\end{array}$ \\
\hline $1970-1979$ & $\begin{array}{l}\text { Atenção voltada aos problemas ambientais, constatação de níveis alarmantes de infecção hospitalar, } \\
\text { transferidas de forma indireta nos RSS. Destaca-se a utilização de técnicas mais avançadas na abordagem } \\
\text { dos problemas decorrentes do uso generalizado de materiais radioativos, da contaminação atmosférica } \\
\text { e da utilização crescente de materiais descartáveis (vantagens, desvantagens, locais para } \\
\text { armazenamento). }\end{array}$ \\
\hline 1980-1989 & $\begin{array}{l}\text { Surgimento da AIDS e consequente perigo de contração da doença por meio de seringas contaminadas. } \\
\text { Vários trabalhos relatam a falta de normas técnicas ou legislação específica capaz de uniformizar os } \\
\text { conceitos e a linguagem, direcionar as ações básicas, definir a melhor forma de gerenciamento, } \\
\text { responsabilidades e a necessidade treinamento/educação dos envolvidos no processo de limpeza. }\end{array}$ \\
\hline 1990-1999 & $\begin{array}{l}\text { Estabelecimento das primeiras normas técnicas e legislações relacionadas aos RSS, porém com pouca ou } \\
\text { ausência de adesão por parte dos estabelecimentos de saúde. Diversos trabalhos são realizados } \\
\text { avaliando-se as etapas do manejo dos resíduos nos estabelecimentos (caracterização física, segregação, } \\
\text { acondicionamento), apresentando-se propostas de gerenciamento tendo em vista a saúde dos usuários } \\
\text { do sistema, os trabalhadores e o ambiente em vista da precariedade das condições identificadas. Com } \\
\text { relação ao tratamento e disposição final, encontram-se vários hospitais com unidades de incineração ou } \\
\text { queima de seus resíduos, porém muitos operando de modo precário, ocasionando poluição atmosférica } \\
\text { e incômoda à vizinhança. }\end{array}$ \\
\hline $2000-2009$ & $\begin{array}{l}\text { Evolução da legislação ambiental com inclusão dos princípios do poluidor pagador, responsabilidade } \\
\text { compartilhada e prevenção à poluição (redução, reutilização e reciclagem). Amplia-se o controle } \\
\text { ambiental às fontes poluidoras, determinando a obrigatoriedade de licenciamento ambiental dos } \\
\text { sistemas de tratamento e disposição final dos RSS. Os estabelecimentos de saúde são obrigados a } \\
\text { apresentar o PGRSS e diversos trabalhos de avaliação dos planos são realizados. Tramitação e aprovação } \\
\text { de políticas públicas relacionadas aos resíduos sólidos. }\end{array}$ \\
\hline
\end{tabular}


Na legislação brasileira, os resíduos sólidos, começaram a ser destacados em legislações federais a partir de 1954, com a Lei Federal 2.312, que trata das Normas gerais sobre defesa e proteção a saúde. Em 1961 o Código Nacional de Saúde regulamentou esta lei federal. Os RSSS, por sua vez, foram disciplinados, por meio da Portaria número 53 de 1979, essa portaria tentou disciplinar, de forma resumida, todo resíduo sólido urbano, provenientes de todas as atividades humanas.

Em 1981 com a Lei federal no 6.938 dispõe sobre a Política Nacional do Meio Ambiente, uma espécie de marco legal para todas as políticas públicas de meio ambiente a serem desenvolvidas por todos os Estados. Com a promulgação da Constituição Federal em 1988, foram estabelecidos diversos direitos de cidadania e impulsionado a participação e a descentralização, principalmente no que se refere à saúde, meio ambiente, direitos da criança e outros.

Alguns anos depois, foi promulgada a Política Nacional de Saúde, por meio da Lei Federal no 8.080, de 19 de setembro de 1990, a qual dispõe sobre as condições para a promoção, a proteção e a recuperação da saúde, revogando a Lei Federal 2.312 e consequentemente o Código Nacional de Saúde.

Em 1993, o CONAMA aprovou a Resolução no05/1993, que estabelece normas mínimas para o tratamento de resíduos sólidos gerados em portos, aeroportos, terminais ferroviários e pelos estabelecimentos prestadores de serviços da saúde. Em 1998 é sancionada a lei no 9.605, a lei de crimes ambientais e em 1999 o decreto no 3.179, regulamento a lei de crimes ambientais.

No ano de 2001, é editada a Resolução CONAMA no283. Esta resolução complementa alguns aspectos referentes ao gerenciamento de RSS não abordados pela resolução CONAMA no 05/1993 e dispõe sobre o tratamento e a destinação final dos resíduos de serviços de saúde; define com maior clareza os RSS e sugere o princípio da minimização na elaboração do Plano de Gerenciamento de Resíduos de Serviços de Saúde (PGRSS).

Em 2003, a Resolução RDC no33 da Agência Nacional de Vigilância Sanitária - ANVISA - aprova o regulamento técnico para o gerenciamento de RSS; define os órgãos de divulgação, orientação e fiscalização da Resolução; determina prazo para adequação ao regulamento técnico dos serviços em funcionamento; define geradores de RSS, estabelece diretrizes de manejo.

A Resolução RDC n³06/2004 da Agência Nacional de Vigilância Sanitária -ANVISA - revoga a RDC n³3/2003 e busca harmonizar as normas federais dos Ministérios do Meio Ambiente por meio do Conselho Nacional de Meio Ambiente/CONAMA e da Saúde através da Agência Nacional de Vigilância Sanitária/ANVISA, referentes ao gerenciamento de RSS. Esta resolução estabelece a classificação dos RSS em cinco grupos, as etapas de manejo, responsabilidades e determina a necessidade da apresentação do PGRSS.

A Resolução CONAMA no358, de 2005, revogou, a Resolução CONAMA no 5, de 1993 a Resolução CONAMA no 283, de 2001. Estabelece a necessidade de apresentação de documento de responsabilidade técnica pelo responsável pelo PGRSS no estabelecimento gerador assim como a necessidade de licenciamento ambiental para os sistemas de tratamento e disposição final de resíduos de serviços de saúde. Estabelece ainda o tratamento e destinação finais adequados às classes específicas de resíduos e ressalta a possibilidade de processos de reutilização, recuperação ou reciclagem. 
Cabe ressaltar que quando se aborda o tema falamos em legislação para gestão de RSSS, há atuação forte de três ministérios, Ministério da Saúde por meio da RDC ANVISA no 306/04 que concentra sua regulação no controle dos processos de segregação, acondicionamento, armazenamento, transporte, tratamento e disposição final e estabelece procedimentos operacionais em função dos riscos envolvidos e concentra seu controle na inspeção dos serviços de saúde, o Ministério do Meio Ambiente com a Resolução CONAMA no 358/05 que trata do gerenciamento sob o prisma da preservação dos recursos naturais e do meio ambiente e estabelece a competência dos órgãos ambientais estaduais e municipais na definição dos critérios para o licenciamento ambiental dos estabelecimentos de Atenção à Saúde e que inclui a necessidade de planos de gerenciamento de resíduos de serviço de saúde conforme a Lei no 12.305/10, que institui a Política Nacional de Resíduos Sólidos (PNRS) e o Ministério do Trabalho com a Norma Regulamentadora 32 de 2005 , que controla os riscos ocupacionais pertinentes às atividades relacionadas à saúde do profissional da saúde, traz em relação aos RSSS que na gestão de resíduo, não basta apenas classificar o resíduo, há a necessidade do dimensionamento de vários parâmetros específicos para cada resíduo estudado pensando na correta segregação, acondicionamento, transporte, tratamento e destinação final. Como os RSSS são um dos maiores responsáveis pelos acidentes em estabelecimentos de saúde, principalmente os perfuro cortantes, a NR 32, traz em seu texto a responsabilidade do profissional de saúde pela segregação dos RSSS e aos estabelecimentos, a obrigação de manter o profissional treinado e capacitado para essa manipulação.

Apesar de a temática dos RSSS ser transversal e interdisciplinar, pois reuni preceitos de saúde ocupacional, saúde pública e meio ambiente, a gestão dos resíduos de serviço de saúde ao ser submetida a legislações de órgãos ministeriais distintos, por muito tempo tem gerado mais conflito e confusão do que efetividade e integração. O alinhamento das legislações, resoluções e normas técnicas iniciou a partir da edição da RDC no 306/2004 (ANVISA), seguida pela Resolução CONAMA no 358/2005 e NR 32/2005. Mas o fato de terem elaborado duas resoluções, da ANVISA e do CONAMA, com tanta similaridade como essas duas com cerca de um ano de diferença por ministérios diferentes, ainda deixa a mostra um descompasso entre estes dois importantes gestores na problemática do RSSS.

\section{Os Resíduos Sólidos dos Serviços de Saúde (RSSS)}

No Brasil, os RSSS foram definidos como todos aqueles que resultam de atividades exercidas nos serviços que têm relação com o atendimento à saúde, tanto humana quanto animal, o que inclui os serviços de assistência domiciliar e de trabalhos de campo; laboratórios analíticos de produtos para saúde; necrotérios, funerárias, serviços de embalsamamento, medicina legal; drogarias e farmácias, estabelecimentos de ensino e pesquisa que abrangem a área de saúde; centros de controle de zoonoses; distribuidores de produtos farmacêuticos; importadores, distribuidores e produtores de materiais e controles para diagnóstico in vitro; unidades móveis de atendimento à saúde; serviços de acupuntura, tatuagem e outros similares (BRASIL, 2005).

Os RSSS são gerados nas etapas de atendimento, cuidado, diagnóstico e tratamento de pacientes em estabelecimentos tais como hospitais, farmácias, drogarias, clínicas veterinárias, consultórios médicos, 
clínicas odontológicas, ambulatórios, unidades básicas de saúde, laboratórios de análises clínicas e patológicas, centros de hemoterapia, unidades de hemodiálise e em centros de pesquisa biomédica (SCHNEIDER E EMMERICH, 2015)

Os RSSS, apesar de representarem uma pequena parcela da totalidade de resíduos sólidos gerados no meio urbano, oferecem um preocupante risco sanitário e ambiental perante um gerenciamento inadequado, pois são possíveis fontes de propagação de doenças, que podem contribuir para o aumento da incidência de infecção hospitalar, além de apresentarem um risco ocupacional dentro e fora dos estabelecimentos de saúde, principalmente em relação aos RSSS do grupo E, quando acondicionado de maneira incorreta (SALES et al., 2009).

Alguns fatores têm contribuído para o aumento da geração de resíduos de serviços de saúde nos países desenvolvidos, como o contínuo incremento da complexidade da atenção à saúde, o uso crescente de material descartável, além do aumento da população idosa que normalmente necessita de mais serviços de saúde sendo usuária com mais frequência de diversos tipos e níveis de especialidades bem como, Unidades de Pronto Atendimento (UPA), Estratégia de Saúde da Família (ESF), Ambulatórios, Hospitais, Policlínicas (ALLEVATO, 2014).

\section{A Problemática do Gerenciamento Inadequado}

A medicina somente tornou-se uma prática hospitalar no século XVIII. O hospital até esta época era um local de acúmulo de doentes, ou seja, era um foco de doenças que causavam efeitos negativos à saúde e ao meio ambiente. Alguns estudos começam a ser desenvolvidos com o objetivo de avaliar as funcionalidades do hospital sob o ponto de vista de seus impactos, inicia-se a preocupação com o hospital em relação ao espaço que está situado e sua ligação com a cidade. O hospital precisa ser localizado em um espaço que não seja favorável à propagação de "miasmas, ar poluído e água suja" (DIAS, 2004).

Além de reunir um grande e variado número de portadores de doenças, o hospital gera um volume de resíduos que são considerados perigosos à saúde e ao meio ambiente, portanto a implantação de ações que minimizem estes impactos é fundamental (DIAS, 2004).

Os resíduos sólidos de serviços de saúde - RSSS, quando gerenciados inadequadamente, oferecem risco ao ambiente, à vida, por suas características biológicas, químicas e físicas. Portanto, implantar políticas de gerenciamento de resíduos nos diversos estabelecimentos de saúde, torna-se fundamental, tendo em vista a promoção da saúde e a qualidade de vida do ambiente (CORREA, et al., 2007).

Ampliando as discussões sobre os riscos associados aos RSSS, trabalhos científicos confirmam o reconhecimento dos riscos desses resíduos pela sobrevivência de agentes dotados de elevada resistência às condições ambientais. Morel e Bertussi Filho (1997) identificaram importantes patógenos nos resíduos sólidos. Nessa pesquisa, a Mycobacterium tuberculosis apresentou um tempo de resistência ambiental de até 180 dias na massa de resíduos sólidos. De forma a destacar outras características peculiares de agentes biológicos na presença de RSS, outro recente estudo, realizado pela Associação de Pesquisa Japonesa, apresentando o estágio ambiental de patógenos nesses resíduos, demonstrou a possibilidade de 
sobrevivência da dose infectante do vírus da Hepatite B ou C, durante uma semana, em uma gota de sangue retirada de uma agulha hipodérmica (NAIME et al., 2007).

Em serviços de saúde, especificamente, o grande aumento de demanda verificado desde a implantação do Sistema Único de Saúde (SUS) no Brasil, que não foi acompanhado por dotações orçamentárias do mesmo porte e que, portanto, geraram uma defasagem na administração global do sistema, e a crescente utilização de materiais descartáveis como forma de controle mais eficiente das infecções e outros fatores associados, tem contribuído decisivamente para o aumento da geração de resíduos (NAIME et al., 2007).

Esta realidade pode ocasionar grandes prejuízos não só financeiros, mas também de riscos a saúde pública, pois quanto maior o volume de resíduos infectantes circulando para os locais de tratamento e disposição final maiores serão as probabilidades de ocorrerem acidentes e consequentes contaminações no ambiente.

A segregação de RSS costuma ser um ponto crítico do processo da minimização de resíduos potencialmente infectantes, podendo trazer resultados insatisfatórios na gestão desses. Sem uma segregação adequada, cerca de 70 a $80 \%$ dos resíduos gerados em serviços de saúde que não apresentam risco acabam potencialmente contaminados. É fundamental coibir a prática de misturar resíduos de áreas com riscos distintos e passar a considerá-los "resíduos infectantes". Essa conduta de misturar resíduos pode ser explicada por razões culturais, operacionais, econômicas, tecnológicas e de recursos humanos (Brasil, 2006).

\section{Gerenciamentos dos Resíduos Sólidos dos Serviços de Saúde na ótica da Saúde Ambiental}

Dentro de uma instituição de saúde, várias são as dimensões da questão ambiental, todas elas muito importantes, complexas e dignas de tratamento sério e sistêmico em seu conjunto. No entanto, é inegável a emergência e a criticidade da gestão dos resíduos hospitalares ou resíduos sólidos dos serviços de saúde (RSSS). Entre as principais causas do crescimento da geração de resíduos dos serviços de saúde, está o contínuo incremento da complexidade dos procedimentos e a universalização do sistema (NAIME et al., 2008).

Sendo assim, o gerenciamento de Resíduos de Serviços de Saúde (RSS) constitui um assunto que merece de uma discussão mais ampliada, tendo em vista as repercussões que pode ter sobre a saúde humana e o meio ambiente. Gerenciar os resíduos de serviços de saúde constitui um conjunto de procedimentos de gestão, planejados e implementados em bases científicas e técnicas, normativas e legais, visando minimizar a produção e proporcionar aos resíduos gerados um encaminhamento seguro e de forma eficiente.

Com base nas características, na classificação dos grupos e no volume gerado, as instituições hospitalares têm como obrigatoriedade a elaboração de um Plano de Gerenciamento de Resíduos de Serviços de Saúde (PGRSS), que estabeleça diretrizes de manejo desses resíduos e contemple a segregação, o acondicionamento, a identificação, o transporte interno, o armazenamento intermediário, o armazenamento 
temporário, o tratamento, o armazenamento externo, a coleta e o transporte externos e a destinação final (SISINNO e MOREIRA, 2005).

Gerenciar adequadamente todo este processo identificando os melhores parceiros e garantindo o reaproveitamento do material é sem dúvida uma das principais tarefas no contexto do manejo dos resíduos (NAIME et al. 2008).

Esta tarefa toma proporções elevadas quando extrapolamos estes cuidados para todos os grupos de resíduos e as especificidades de cada um, pois, conforme a resolução 306/04 da ANVISA existe um conjunto de procedimentos a ser adotado conforme o tipo de resíduo gerado e o gerador é o responsável por todas as etapas do manejo inclusive a disposição final, portanto a empresa deverá acompanhar e garantir que os resíduos receberão tratamento adequado (NAIME et al. 2008).

A preocupação como gerenciamento dos Resíduos de Serviços de Saúde (RSS) é algo recente dentro das instituições de saúde e somente passou a ganhar devida importância com a aplicação de legislações específicas. As resoluções 306/04 da Agência Nacional de Vigilância Sanitária (ANVISA) e 358/05 do Conselho Nacional do Meio Ambiente (CONAMA) dispõem sobre o regulamento técnico para o gerenciamento de resíduos de serviços de saúde e tornam obrigatória a qualificação dos profissionais que atuam com tais resíduos.

Farias (2005), em sua pesquisa, ressalta que uma vez adotada a classificação dos RSSS é possível identificar aspectos qualitativos e quantitativos das diferentes frações geradas nos estabelecimentos de saúde, considerando dentre outros fatores, a fonte de geração e a periculosidade do resíduo. Acrescenta ainda, que essas considerações contribuem para que no gerenciamento, seja definido com critério o tipo de tratamento e disposição final para cada classe de resíduo, e a possibilidade da minimização de riscos aos trabalhadores que manuseiam tais resíduos, intra e extra-estabelecimentos de saúde e ao ambiente.

O gerenciamento dos resíduos de saúde tem como objetivo minimizar a produção dos mesmos e proporcionar um encaminhamento seguro, visando à proteção dos trabalhadores e a preservação do ambiente. No que se refere às condições de trabalho, a estrutura e a organização estão relacionadas à sua divisão técnica, ao processo e ao ritmo de trabalho, à distribuição de atividades entre os profissionais, aos níveis de formação e especialização do trabalho. Dependendo da forma como a instituição está estruturada e organizada internamente, os profissionais terão melhores ou piores condições de trabalho. Sendo assim, a forma como a instituição lida com a segregação de resíduos passa a ser de extrema importância para a saúde do trabalhador, esteja ele ligado diretamente à assistência ou não (MACEDO et al., 2007).

A complexidade do gerenciamento dos RSSS exige uma análise inter e transdisciplinar e o conhecimento sobre variáveis relacionadas com diferentes áreas do conhecimento. A instituição, ao definir suas políticas de gerenciamento, precisa analisar não apenas as variáveis internas que determinam a geração dos RSSS, mas o conjunto de relações das variáveis externas que acaba por interferir nos resultados que podem ser obtidos. Isto aliado a programas educativos, capacitações constantes, pesquisas e análise de indicadores, que envolvam a instituição como um todo, constitui fatores fundamentais para a efetivação de programas de gerenciamento (SCHNEIDER, 2004). 


\section{CONSIDERAÇÕES FINAIS}

Dentro do contexto estudado sobre os resíduos sólidos dos serviços de saúde, a questão mais discutida é o gerenciamento do RSSS, levantando a necessidade da conscientização dos profissionais para o cuidado com a segregação dos resíduos gerados durante sua atuação nos serviços de saúde. Vários estudos apontam a expectativa de que profissionais de todos os níveis e áreas de atuação, sejam conscientes da importância da correta segregação dos resíduos gerados nos serviços de saúde, sejam mais atuantes no processo, encaminhando apenas para tratamento aqueles materiais que realmente necessitem ser tratados.

Os resultados desta análise teórica e bibliográfica apontam para a necessidade de implantar políticas de gerenciamento dos resíduos sólidos de serviços de saúde (RSSS) nos diversos estabelecimentos de saúde, não apenas investindo na organização e sistematização dessas fontes geradoras, mas, fundamentalmente, mediante o despertar uma consciência humana e coletiva quanto à responsabilidade com a própria vida humana e com o ambiente.

Assim é necessário da observância rigorosa do gerenciamento do RSSS, devido ao potencial infeccioso degradante e poluente contra o meio ambiente e infeccioso contra a saúde humana, os resíduos de serviços de saúde exigem atenção especial e técnicas corretas de manejo e gerenciamento. Isto envolve desde a etapa de geração até o momento de disposição final.

O gerenciamento correto é extremamente necessário e importante para a garantia e segurança dos trabalhadores da área da saúde, da área da limpeza, da coleta de resíduos, além da proteção da comunidade e do meio ambiente.

\section{REFERÊNCIAS}

ALLEVATO, C. G.. Resíduos de serviços de saúde: o conhecimento dos profissionais que atuam no contexto hospitalar. Dissertação (Mestrado em Enfermagem. Universidade Federal do Estado do Rio de Janeiro, Rio de Janeiro, 2014.

BRASIL. Agência Nacional de Vigilância Sanitária (BR). RDC no 306, de 7 de dezembro de 2004: dispõe sobre o Regulamento Técnico para o gerenciamento de resíduos de serviços de saúde. Brasília, 2004.

BRASIL, Conama. Conselho Nacional do Meio Ambiente (BR). Resolução no 358, de 29 de abril de 2005: dispõe sobre o tratamento e a disposição final dos resíduos dos serviços de saúde e dá outras providências. Brasília, 2005.

BRASIL. Decreto no 5.940, de 25 de outubro de 2006. Institui a separação dos resíduos recicláveis pelos órgãos e entidades da administração pública federal direta e indireta., Brasília, 2006

CAMPONOGARA, S.; KIRCHHOF, A. L. C; RAMOS, F. R. S.. Uma revisão sistemática sobre a produção científica com ênfase na relação entre saúde e meio ambiente. Ciência e Saúde Coletiva, Rio de Janeiro, v. 13, n. 2, p. 427-439, 2008.

CAMPOS, F. A.; OLIVEIRA, F. J. G.; FROTA, N. M.; CAETANO, J. A.; ORIA, M. O. B.; PINHEIRO, P. N. C.. Avaliação do conhecimento dos profissionais do bloco cirúrgico quanto ao gerenciamento dos resíduos sólidos em saúde. Vigilância Sanitária em Debate: Sociedade, Ciência e Tecnologia, v.2, n.1, p.33-38, 2014.

CARVALHO, R. F.. Avaliação do Gerenciamento de Resíduos dos Serviços de Saúde: Estudo de Caso do Hospital Municipal Dr. Mário Gatti. In: Congresso Brasileiro de Gestão Ambiental, Bauru - SP, 2010.

CORREA, L. B.; LUNARDI, V. L.; CONTO, S. M.; GALIAZZI, M. C.. O saber resíduos sólidos de serviços de saúde na formação acadêmica: uma contribuição da educação ambiental. Interface, Botucatu, v.9, n.18, p.571-584, 2005.

CORREA, L. B.; LUNARDI, V. L.; CONTO, S. M.. O processo de formação em saúde: o saber resíduos sólidos de serviços de saúde em vivências práticas. Revista. Brasileira de Enfermagem, Brasília, v.60, n.1, p.21-25, 2007.

DIAS, M. M. A.. Resíduos de serviços de saúde e a contribuição do hospital para a preservação do meio ambiente. Revista Acadêmica de Enfermagem. Academia Brasileira de Especialistas em Enfermagem - ABESE. v.2. n.2, 2004. São Paulo: Demais Editora. 2004.

FARIAS, L. M. M. Impasses e possibilidades do gerenciamento de resíduos de serviços de saúde no Brasil: 
um estudo de caso no Centro de Saúde Escola Germano Sinval Faria - ENSP/Fiocruz. Dissertação (Mestrado em Saúde Pública) - Escola Nacional de Saúde Pública, Rio de Janeiro, 2005.

LÜDKE, M.; ANDRÉ, M. E. D. A.. Evolução da Pesquisa. In: LÜDKE, M.; ANDRÉ, M. E. D. A.. Pesquisa em educação: abordagens qualitativas. 2 ed. São Paulo: EPU, 2003.

LUZ, F. X. R.; GUIMARÃES, C.. Resíduos hospitalares. Rev. Saúde Pública, São Paulo, v.6, p.405-26, 1972.

MACEDO, L. C.; LAROCCA, L. M.; CHAVES, M. M. N.; PERNA, P. O.; MUNTSCH, S. M. A.; DAMACENO, E. F. C.; SOUZA, T. S.; POLIQUESI, C. B.; TRUPPEL, T. C.; SOUZA, C.. Segregação de resíduos nos serviços de saúde-a educação ambiental em um hospital-escola. Cogitare Enfermagem, Curitiba, v.12, n.2, p.183-188, 2007.

MINAYO, M. C. S.. Pesquisa social: teoria, método e criatividade. 23 ed. Petrópolis: Vozes, 2004.

MOREL, M. M. O.; BERTUSSI FILHO, L. A.. Resíduos de Serviços de Saúde. In: RODRIGUES, E. A. C.. Infecções Hospitalares: prevenção e controle. São Paulo: Sarvier, 1997.

NAIME, R.; RAMALHO, A. H. P.; NAIME, I. S.. Diagnóstico do Sistema de Gestão dos Resíduos Sólidos do Hospital de Clínicas de Porto Alegre. Estudos tecnológicos, São Leopoldo, v.3, n.1, p.12-36, 2007.

NAIME, R.; RAMALHO, A. H. P.; NAIME, I. S.. Avaliação do sistema de gestão dos resíduos sólidos do hospital de clínicas de Porto Alegre. Revista Espaço para a Saúde, Londrina, v.9, n.1, p.1-17, 2008

PUGLIESI, E. Estudo da evolução da composição dos resíduos de serviços de saúde (RSS) e dos procedimentos adotados para o seu gerenciamento integrado, no Hospital Irmandade Santa Casa de Misericórdia de São Carlos-SP. Tese (Doutorado em Ciências da Engenharia Ambiental) Universidade de São Paulo, São Carlos, 2010.
RAMÍREZ, M. C. T.. Plano de gerenciamento de resíduos de serviços de saúde: proposta de modelo para um hospital do Município do Panamá, República do Panamá. Dissertação (Mestrado em Saúde Pública) - Universidade de São Paulo, São Paulo, 2012.

RAMOS, D. A. B.. Impasses e dificuldades na gestão de resíduos de serviços de saúde em unidades básicas de saúde: estudo de caso no município de Araçatuba - SP. Dissertação (Mestrado em Saúde Pública) - Escola Nacional de Saúde Pública Sergio Arouca, Rio de Janeiro, 2013.

RISSO, W. M.. Gerenciamento de Resíduos de Serviços de Saúde: a caracterização como instrumento básico para abordagem do problema. Dissertação (Mestrado em Saúde Ambiental) - Universidade de São Paulo, São Paulo, 1993.

SALES, C. C. L.; SPOLTI, G. P.; LOPES, M. S. B; LOPES, D .F.. Gerenciamento dos resíduos sólidos dos serviços de saúde: aspectos do manejo interno no município de Marituba, Pará, Brasil. Ciênc. saúde coletiva, Rio de Janeiro, v.14, n.6, p.2231-2238, 2009.

SCHNEIDER, V. E.. Sistemas de gerenciamento de resíduos sólidos de serviços de saúde: contribuição ao estudo das variáveis que interferem no processo de implantação, monitoramento e custos decorrentes. Porto Alegre. Tese (Doutorado em Engenharia de Recursos Hídricos e Saneamento Ambiental) - Universidade Federal do Rio Grande do Sul, 2004.

SCHNEIDER, V. E., EMMERICH, R. C. P.. Resíduos de serviços de saúde. IN: Resíduos de serviços de saúde: um olhar interdisciplinar sobre o fenômeno: organizadores. Caxias do Sul: EDUCS, 2015.

SISINNO, C. L. S.; MOREIRA, J. C.. Ecoeficiência: um instrumento para a redução da geração de resíduos e desperdícios em estabelecimentos de saúde. Caderno de Saúde Pública, Rio de Janeiro, v.6, n.21, p.1893-1900, 2005. 